\title{
La Contratación Pública Y Su Incidencia En El Fomento De La Producción De Las PYMES Manufactureras De La Provincia Del Guayas- Ecuador
}

\section{Ing. Com. Eliana Valeria Villegas Acevedo Erika Jadira Romero Cárdenas}

Docente Facultad de Ciencias Administrativas y Comerciales

Universidad Estatal de Milagro

Estudios Realizados: Universidad De Guayaquil - Economista 2003;

Universidad Tecnológica Empresarial Gquil.-Magíster Administración Y

Dirección De Empresas 2008

Experiencia Docente: Catedrática De La Universidad Estatal De Milagro Y

Universidad Nacional San Marcos De Lima-Doctorado En Ciencias

Administrativas

Diseño Curricular Por Competencia Universidad De Guayaquil 2008

URL:http://dx.doi.org/10.19044/esj.2019.v15n8p213

\section{Resumen}

Este documento se centra en el análisis del proceso de adquisición en el sector manufacturero de la provincia de Guayas. La metodología utilizada en este estudio se basa en un enfoque longitudinal de un enfoque cuantitativo, no experimental. Además, se trata de un estudio exploratorio descriptivo. La hermenéutica es una de las técnicas utilizadas para la recolección de datos. Permite una revisión bibliográfica de estudios por adelantado, agregando la triangulación de ideas basada en la evidencia. El resultado fue una claridad simétrica entre el mercado público y su crecimiento para las empresas que ingresan a su entorno. Este estudio se basa en un promedio de 32.76. Sin embargo, la desviación estándar es de 4.816. Por esta razón, las políticas públicas deben centrarse en mejorar el acceso a la información para los proveedores y el público en general, así como en fortalecer los mecanismos de control institucional y social.

Palabras clave: Optimizar, Procesos, Contratación pública, Estado, Administración 


\title{
Public Procurement and its Incidence in the Promotion of the Production of Manufacturing SMEs of the Province of Guayas-Ecuador
}

\author{
Ing. Com. Eliana Valeria Villegas Acevedo \\ Erika Jadira Romero Cárdenas
}

Docente Facultad de Ciencias Administrativas y Comerciales

Universidad Estatal de Milagro

Estudios Realizados: Universidad De Guayaquil - Economista 2003;

Universidad Tecnológica Empresarial Gquil.-Magíster Administración Y

Dirección De Empresas 2008

Experiencia Docente: Catedrática De La Universidad Estatal De Milagro Y

Universidad Nacional San Marcos De Lima-Doctorado En Ciencias

Administrativas

Diseño Curricular Por Competencia Universidad De Guayaquil 2008

\begin{abstract}
This paper focuses on the analysis of the public procurement with the purpose of establishing the incident factors in the production of the manufacturing SMEs of the province of Guayas. The methodology used in the study is based on a longitudinal cut design with a quantitative approach, of a non-experimental nature. In addition, it is an exploratory-descriptive study. Among the techniques used for data collection is hermeneutics, which allows a documentary analysis of studies carried out in advance, adding to it the triangulation of ideas to emit criteria according to the evidence. As a result, there was a symmetrical clearness between the public procurement and its growth to the companies that enter their environment. This was observed in the sample of 80 SMEs with an average of 32.76. On the other hand, the standard deviation is 4.816. For this reason, public policies should focus on improving access to information by suppliers and the general public, as well as strengthening the institutionality and mechanisms of social control.
\end{abstract}

Keywords: Optimize, Processes, public Procurement, State, Administration

\section{Introduction}

El desarrollo del presente análisis tiene como objetivo principal determinar el nivel de incidencia de la Contratación Pública en el fomento productivo de las Pymes manufactureras de la provincia del Guayas. Para lo cual, se efectuó la revisión de datos estadísticos proporcionados por el Servicio Nacional de Contratación Pública (SERCOP), por considerarse la 
organización encargada de liderar y regular la transparencia y efectividad de la contratación pública en las diferentes áreas de producción.

En este sentido, Ibarra Cisneros, González Torres, y Dummer Flores (2017) en su artículo competitividad empresarial de las pequeñas y medianas empresas manufactureras de Baja California, expresan la importancia que radica la competitividad de las Pymes pertenecientes a este campo productivo en el tejido industrial del Estado. Además, de considerarlas fuentes generadoras de empleo que contribuyen al dinamismo de la economía local.

Por esta razón, las Pymes se proyectan como organizaciones dominantes en gran parte de los países del mundo, lo que representa el 95\% de los negocios existentes en el mercado productivo internacional, las mismas que cuentan con un sinnúmero de ventajas competitivas con respecto a las grandes empresas, haciendo énfasis en su menor tamaño y facilidad para adaptarse a los cambios en la economía y patrones de oferta-demanda (González, 2013).

Vázquez y Arredondo (2014), en su estudio Importancia de las pymes en el mundo, asegura que en la actualidad las pequeñas y medianas empresas se consideran como factor dominante en el fortalecimiento de la economía, debido a su actividad productiva propicia la creación de nuevas plazas de empleo, situación que contribuye a la satisfacción de las necesidades básicas de los hogares, así como al desarrollo social de un país.

De acuerdo al informe de ProMéxico (2013) en su estudio, Pymes, eslabón fundamental para el crecimiento en México, demuestra como las Pymes son consideradas como la columna vertebral de la economía mexicana, donde los acuerdos comerciales propiciados por las políticas gubernamentales han favorecido los ámbitos productivos a través de la contratación pública durante los últimos años, sumándose a ello su alto impacto en los niveles de producción nacional.

Debido a esto, las pymes manufactureras aportan con el 50\% de las exportaciones en México. Según Rosagel (2012) “el estado mexicano reportó alrededor de 2500 millones de dólares en exportación productiva por parte de las pymes en el período 2011, donde la mitad provenía del sector manufacturero destinado a proveer el mercado doméstico” (p.80).

En lo referente a Ecuador, Yance, Solís, Burgos, y Hermidia (2017) en su artículo la importancia de las Pymes en el Ecuador, manifiesta que "las pequeñas y medianas empresas en el país constituyen un factor importante en el crecimiento socio-económico, lo que requiere de la implementación de estrategias que beneficien en gran medida las operaciones, con la finalidad centrada en la reducción de los costos de operación” (p.89).

Ante lo manifestado, es evidente que las Pymes en el Ecuador contribuyen a la generación de empleo, por lo que resulta prioritario que logren alcanzar su desarrollo sostenible en el tiempo como resultado de la 
optimización de procesos y productos de calidad, sumándose a ello, su participación activa en la Contratación Pública (CP).

Para el Instituto Nacional de Estadísticas y Censos-INEC (2010) "las pymes en el contexto ecuatoriano se caracterizan por el uso intensivo de la mano de obra, escasa oportunidad para la implementación de la tecnología, reducción de capital, baja productividad y escasa participación en las contrataciones estatales” (p.89).

Según el estudio realizado por la CEPAL (2011) sobre la situación de las Pymes en América Latina demuestra que, Ecuador ocupa la mayor proporción de las pequeñas y medianas empresas en la región con el 44\%, además de favorece a la generación de empleo en un 24\% y el 15,9\% de las ventas.

En lo referente a la productividad de las Pymes, éstas permiten dinamizar la economía del país por su nivel de participación en el mercado de ofertas públicas. Sin embargo, existen situaciones que demuestran estados de inequidad o monopolio en la consignación de los contratos públicos orientados a diversas regiones o empresas específicas que termina deteriorando el modelo productivo y de economía solidaria de las mismas.

En este sentido, cada uno de los estudios citados han evidenciado la importancia de las Pymes en el dinamismo de la economía de los países, por lo que el abordaje del tema "La contratación pública y su incidencia en el fomento de la producción de las pymes manufactureras de la provincia del Guayas-Ecuador”, se ha orientado en un proceso investigativo documental que contrasta su desarrollo y crecimiento en base a la información proporcionada por los diversos organismos de control, considerando que la indagación se justifica por la presencia de factores ligados a las políticas públicas, tales como montos adjudicados y participación del número de empresas tomando en consideración su zona geográfica, en el caso de la provincia del Guayas, así como el sector productivo referente a las pymes manufactureras.

Por tanto, el análisis contenido en este artículo demuestra los niveles de incidencia de los parámetros de igualdad, equidad y confianza que deben existir en la contratación pública, con la finalidad de generar expectativas y motivación a empresarios, induciéndolos a participar en licitaciones relacionadas a las empresas públicas, donde el criterio de los pequeños productores referente a su desistimiento se ajustan al retraso en los pagos y preferencias de para ciertos proveedores, situación que se contrapone a los principios que versan en la CP y su Reglamento de aplicación, respecto a la satisfacción de necesidades y del bien común sin discriminación alguna.

Además, las compras públicas y la forma en que se llevan a cabo pueden tener múltiples implicaciones económicas, sociales y ambientales. En efecto, las adquisiciones gubernamentales pueden ser utilizadas como herramienta de política para promover ciertos objetivos en materia económica, 
estimulando determinados tipos de empresa y sectores considerados estratégicos, además del desarrollo de economías regionales y locales en plena relación con el ambiental, facilitando procesos productivos consientes en la sostenibilidad ambiental y de manera social desincentivando el trabajo informal o facilitando la inserción laboral de grupos desfavorecidos.

Durante los últimos años, los contratos públicos eran considerados apenas como una herramienta necesaria para considerar el funcionamiento de las distintas empresas gubernamentales, y la atención se focalizaba meramente en que los procedimientos se adecuaran a las normas legales vigentes y el gasto no superen las asignaciones presupuestarias dispuestas. No obstante, teniendo en cuenta el amplio espectro de sus repercusiones, está claro que el diseño e implementación de un sistema adecuado de compras gubernamentales no puede considerarse simplemente una cuestión técnica: su mejor o peor funcionamiento afectará directamente la calidad de la población. (G Rozenwurcel., L Drewes, 2012)

Desde estas perspectivas, el desarrollo de la temática se ajusta a un criterio de investigación mixta (cuantitativo-cualitativo), donde se pondera una serie de factores incidentes en la Contratación Pública en el país, especialmente entre los pequeños productores de la provincia del Guayas.

\section{OBJETIVOS GENERAL}

- Analizar la contratación pública y su incidencia significativa en la producción de las pymes manufactureras en la provincia del Guayas a través de la revisión documental de imformes prporcionados por los entes de control.

\section{OBJETIVOS ESPECÍFICOS}

- Indagar como la relación de los proveedores incide en la producción en las pymes manufactureras.

- $\quad$ Determinar de qué manera las normativas de contratación pública inciden de manera significativa en la producción de pymes manufactureras de la provincia de la Guayas.

\section{I.}

Desde un contexto general, hoy en día, las Pymes se encuentran distribuidas en el mercado de diferentes formas y dimensiones, sean estas por sociedades o de un solo propietario, las mismas que cuentan con la plena libertad para desarrollar cualquier actividad, entre las que destacan la producción, comercialización o prestación de servicios donde se enfoque su utilidad. Por otra parte, en base al Estudio de Gestión Competitiva de las pequeñas y medianas empresas en el Ecuador, éstas representan el 95\% de las unidades productivas en el país (Jácome \& King, 2013, p. 15). 
Bajo este contexto, es evidente que el Ecuador cuenta con un gran número de Pymes en el área de comercialización, de servicio o industrial, las mismas que contribuyen a la generación de empleos. Debido a ello, éstas afrontan un grado de relevancia en el mercado comercial y laboral del país, además en el desarrollo de la economía. No obstante, presentan algunas dificultades a nivel global como local a lo largo de los años, donde se pueden mencionar los efectos de la globalización, viéndose afectadas por la prolongación de la competitividad mundial, por considerar que gran parte de ellas no cuentan con el apoyo financiero, economías de escala o no alcanzan a ser suficientemente competentes para mantenerse en los mercados de demanda.

Por ello, las pymes ecuatorianas se han visto en la necesidad de acoplarse a la lógica de libre comercio, con la finalidad de reproducir de manera efectiva el capital, lo que deriva en una creciente interconexión de los mercados internacionales; y éstos a su vez, se ven afectados por el nivel de fuerza y velocidad generada por los estados de crisis o eventos negativos del sistema económico a nivel mundial (López \& Martínez, 2012, pág. 58).

Las Pymes en el Ecuador son consideradas como el sector más productivo en la economía, por su impacto que tiene en el desarrollo del país. Debido a ello, su aporte se ve reflejado en el Producto Interno Bruto (PIB) con una tasa promedio que supera el 25\%; y, a su vez, es generadora de empleo que ampara al 70\% de la Población Económicamente Activa (PEA). En este sentido, el $39 \%$ de las plazas laborales son proporcionadas por las microempresas, mientras que el $17 \%$ a pequeñas y el 14\% a medianas (Jácome \& King, 2013, p. 53)

Ante lo mencionado, se destaca la importancia de las pequeñas y medianas empresas como un elemento dinamizador de la economía, la misma que se proyecta como bases para el desarrollo de la producción, es decir que, entre más producción, más demanda y compras de productos, por ello se debe añadir más valor agregado con la finalidad de generar más riqueza y empleo como parte de un manejo eficiente de la economía.

Según el aporte de Paz y Miño (2015, p. 89), durante este período 2011 existieron alrededor de 27.646 Pymes, de las cuales 13.322 se encontraban distribuidas en medianas, lo que representa el 34,96\% y 8.7671 pequeñas empresas que ampara al 65,04\%. De las cuales, la mayor cantidad se encuentran ubicadas en Pichincha y Guayas, con un porcentaje del 43,29\% y $40,46 \%$ respectivamente.

En concordancia con lo manifestado por los autores antes citados, las Pymes manufactureras en el Ecuador son significativas para el desarrollo, la misma que hace referencia al proceso transformador de la materia prima en un producto para su uso final o que sirve como insumo la realización de otros procesos de producción. A nivel nacional, en mayo del 2017, los índices de producción de la Industria Manufacturera (IPI-M) alcanzó un resultado de 
90,01; comparándose con el mes anterior, por lo que se obtiene una variación mensual de 3,60\%, lo que es traducido en un incremento de la producción a nivel nacional (Instituto Nacional de Estadística y Censo, 2017, p. 4).

Desde esta perspectiva, resulta importante tener en claro los conformantes del sector industrial manufacturero, entre los que se encuentran las empresas dedicadas a la elaboración de productos alimenticios, bebidas, tabacos, textiles, prendas de vestir, industria del cuero, madera y sus derivados, artículos de paja, fabricación de papel y sus derivados, sustancias químicas medicinales, productos botánicos, industrias metálicas básicas, fabricación de productos metálicos, maquinaria, equipos, preparación de productos minerales no metálicos, confección de joyas, entre otras, con una participación activa en el desarrollo productivo del país, así como elemento dinamizador de la contratación pública.

Según el análisis del INEC (2012) relativo al comercio minorista en el país, éste se encuentra integrado por 232.760 establecimientos económicos, de un total de 500.217 que declararon actividad económica en el Censo Nacional Económico en el 2010. Por tanto, dentro de las actividades económicas que competen a este margen porcentual, se encuentra la venta de alimentos, bebidas y tabaco con el 50,5\%, otras actividades de comercio al por menor con el 23,3\%, prendas de vestir, calzado, artículos de tocador con el 4,7\%, muebles y equipos de iluminación con el 3,0\%.

Con relación en aquello, la distribución establecida por provincia, deja entrever que gran parte de estos se concentra en la provincia del Guayas con el 25,2\%, seguido por Pichincha y Manabí, de ahí que la necesidad de mejorar la participación del sector manufacturero en los procesos de contratación pública es relevante, considerando que las Pymes asociadas a esta actividad, según el informe presentado por el SERCOP evidenció un porcentaje de participación hasta agosto del 2017 de apenas el 16,7\% disminuyendo alrededor de 7 puntos referentes al año anterior (SERCOP, 2017, p. 16).

Dicha disminución se ve reflejada en el porcentaje de participación por provincia contratante, donde Pichincha se ubica con el $41,4 \%$, seguido del Guayas con el 18,3\%, notándose una diferencia significativa en cifras de participación, que en muchos de los casos se relacionan por la falta de expectativas o referencias negativas del proceso de licitación, asociadas por indicadores de corrupción, desigualdad o falta de incentivos para el sector manufacturero local, especialmente al pequeño productor.

\section{De la Contratación Pública (CP)}

La Ley Orgánica del Sistema Nacional de Contratación Pública, señala en el artículo 4, los principios que se deberán observar en materia de contratación pública: Art. 4.-Principios.-Para la aplicación de esta Ley y de los contratos que de ella se deriven, se observarán los principios de legalidad, trato justo, 
igualdad, calidad, vigencia tecnológica, oportunidad, concurrencia, transparencia, publicidad; $\mathrm{y}$, participación nacional.

Por otra parte, dentro del principio de legalidad, Rivas y López (2010) en su libro Interrogantes y respuestas sobre la Ley de Contratación Pública, manifiesta que:

Las entidades contratantes deben cumplir las disposiciones previstas en la Ley, Reglamento y Resoluciones expedidas por el Instituto Nacional de Contratación Pública y demás normativas expedidas por los órganos competentes, que regule la adquisición y arrendamiento de bienes, la ejecución de obras y la prestación de servicios incluidos los de consultoría, así como para la adecuada administración y control de los recursos públicos (p.24).

En relación a los principios de calidad, Murán (2012) asegura que: “ningún otro aspecto posee gran alcance o vitalidad, como la idoneidad o aptitud para el uso (p.123). Actualmente es denominado como calidad, aplicado de manera prioritaria a los bienes y servicios.

Uno de los aspectos fundamentales a considerar en la economía actual, es el impulso que se le ha dado a la contratación pública en Latinoamérica, avizorando nuevos rumbos a las pequeñas y medianas empresas (PYMES), situación que conlleva a incrementar el interés de realizar estudios sobre el impacto que éste genera sobre ellas.

En el presente acápite se logra una exploración breve de la tendencia internacional respecto a los aspectos que tienden a polemizarlo aspectos generales de la contratación pública, en relación a su grado de apertura y accesibilidad de las pymes. En consecuencia, las contrataciones del estado, actualmente ha cobrado un nivel de significancia máxima debido al volumen de recursos que se involucra en el mismo.

Según González Tamayo (2016), manifiesta que “existe un promedio generalizado entre el 10 al 15\% de recursos involucrados en relación al Producto Interno Bruto (PIB) de los países” (p. 3), situación que pone en manifiesto su gran trascendencia en lo referente al manejo eficiente de los recursos públicos, enfocada siempre al cumplimiento de las finalidades del Estado, enfatizando en la transparencia como valor de carácter social y por ende, a la generación de las interrelaciones económicas entre el gobierno y los ciudadanos, quienes se presentan con la figura de proveedores o agentes suministradores de bienes y/o servicios, contratistas de obras públicas entre otros.

Desde estas perspectivas, el análisis de la Contratación Pública (CP) nos direcciona a establecer una línea de tiempo sobre el surgimiento en las normativas ecuatorianas; tal es así que en el 2008 se logra expedir dos cuerpos normativos de gran relevancia para las dimensiones de la contratación pública, los mismos que tuvieron la capacidad de transformar el esquema jurídico 
procedimental, pero, sobre todo, se logró imponer una visión de carácter sistémica en esta área, incorporando las nuevas tendencias tecnológicas, donde las tecnologías de la Información y Comunicación (TIC) harían su incursión dentro del mercado público.

Ante aquello, la constitución ecuatoriana en su artículo 275 establece a la planificación como un medio eficaz para alcanzar el desarrollo a mediano plazo, debido a esto, el sector público está obligado a cumplirlo; por su parte la Ley Orgánica del Sistema Nacional de Contratación Pública-LOSNCP, instituye a las Entidades del Estadio previo la contratación de un bien o servicio se realice anualmente la planificación de todas sus compras, situación que tiende a evitar improvisaciones, direccionamiento y gastos ineficientes de los recursos del estado, aportando de manera significativa al cumplimiento de los objetivos institucionales y del Plan Nacional de Desarrollo-PNBV (Casagallo, 2016).

Por tanto, la Constitución de la República del Ecuador, incorpora por primera vez una expresa disposición sobre la contratación pública, estimando que: "las compras públicas cumplirán los criterios de eficiencia, transparencia, calidad, responsabilidad ambiental y social. Se priorizará los productos y servicios nacionales, especialmente los provenientes de la economía popular y solidaria, y de las micro, pequeñas y mediana unidades productivas" (Constitución de la República del Ecuador, 2008).

No obstante, ante los criterios de priorización surgen una serie de problemas debido a la evidente ausencia de igualdad, no discriminación o maximización de la competencia, que en muchos de los casos se da de manera deliberada o no, situación que es significativa, porque se vincula a la priorización con la producción nacional.

Cabe mencionar, que uno de los pilares fundamentales de la contratación pública es la eficiencia, situación que se traduce en términos generales en conseguir el mayor valor monetario empleado a los menores costos operativos y transaccionales posibles. Debido a esto, surgen los indicadores de competencia, considerado como uno de los supuestos para alcanzar la eficiencia. Sin embargo, en el sistema de CP vigente en el Ecuador, existen licitaciones donde la concurrencia es mínima, por tanto, se logra minimizar los beneficios, generándose de esta manera privilegios a favor de determinados operadores económicos contraponiéndose al legítimo interés de todos aquellos que se encontraban en capacidad de ofertar bienes y servicios, así como en prejuicio de la colectividad en general. Por esta razón, el Estado en esa instancia logra incurrir en compras costosas y de mala calidad, malgastando los recursos públicos.

Ante aquello, existen supuestos que generalizan la deficiencia o problemática alrededor de la contratación pública en el país, especialmente en el sector manufacturero, asumiendo un difícil acceso a las diversas ofertas o 
licitaciones, sumándose el retraso en los pagos por parte de las instituciones públicas ocasionando en muchos de los casos la inestabilidad de capital que tiende a sucumbir en la quiebra o cierre del negocio.

Además, en el país no se logra consolidar los principios de la Economía Popular y Solidaria, ya que se evidencia la carencia de fuentes de financiamiento para capital de trabajo, que en muchos de los casos se deben a las garantías que exigen las entidades públicas o privadas para la concesión de un préstamo, situación que refleja la incapacidad de las pymes para responder a las demandas de las empresas públicas, dando paso libre a los sectores productivos de mayor financiamiento, situación que va en contra de la igualdad de oportunidades.

Otro de los factores asociados a la problemática radica especialmente en los requisitos exigidos para llevar a efecto la contratación pública, situación condicionante de inestabilidad para el emprendedor, pues su material productivo se encuentra elaborado y no logra ofertarlo a quienes lo requieren, puesto que genera pérdidas económicas que perjudica su estabilidad productiva.

\section{De la producción}

Fernández (2014) considera que, la productividad es definida como "la forma de utilización de los factores de producción en la generación de bienes y servicios para la sociedad” (p.114). Así pues, la productividad se convierte en el objetivo estratégico de las organizaciones, por estimar que, sin ellas los productos y servicios no lograrían alcanzar los niveles de competitividad necesaria dentro de los mercados que incursiona.

Por otra parte, Medina (2012) concibe a la productividad como una estrategia, encargada de buscar los mejores niveles de desempeño de la organización en el mercado globalizado, para permitir que la empresa trabaje de manera consecutiva por alcanzar los objetivos empresariales, como parte del encadenamiento de sus procesos y un óptimo nivel de operación de sus recursos.

Desde una proyección general, se ventila la importancia del segundo pilar de las compras públicas que permite fomentar el desarrollo económico de la comunidad, a lo que se conoce como componente nacional, en el cual se discuten las virtudes y oportunidades que brindan las políticas públicas discriminantes a favor del mismo, como también de los posibles riesgos que impone el establecimiento de requerimientos discriminatorios.

Ante aquello, el componente nacional se logra construir por medio del valor agregado de la actividad económica y del componente nacional en factor costo. Por ende el sector manufacturero tiende a argumentar que la elaboración de su producto gira alrededor de cuatro elementos, que son la mano de obra directa, la materia prima, maquinaria y tecnología, situación incidente en una 
serie de estimaciones para establecer el aporte de este sector a la contratación pública en el país, teniendo en cuenta la base de la declaración de impuesto donde se contiene información del valor de la materia prima, la mano de obra y los servicios profesionales, sumándose a ellos las depreciaciones.

Por ello, la situación de las empresas manufactureras en el país, especialmente en la provincia del Guayas ha sufrido reveses económicos significativos en relación a otros sectores productivos que tienden a incursionar en el sistema de Contratación Pública, haciendo énfasis que este sector productivo se encuentra ligado al sector de la construcción, ya que se requiere de materiales manufactureros para su culminación, muestra de aquello es el acondicionamiento de muebles para oficina, entre otros.

Debido a esto, la presente investigación se justifica por la necesidad de mejorar las políticas públicas en relación a la Contratación Pública, pues existen muchos factores que inciden en la estabilidad económica financiera de las Pymes, especialmente del sector manufacturero, quienes se encuentran ligados o condicionados a terciar en contrataciones de gran relevancia como elementos complementarios que limitan su productividad.

Ante aquello, la relevancia de la investigación se proyecta como un aporte significativo al análisis situacional de la CP, que por tratarse de un modelo estatal no sucumbe a criterios realizados de manera externa, sino a informes propiciados a través de procesos de auditorías internas o de retroalimentación, que en mucho de los casos no ampara criterios de los propios elementos intervinientes, haciendo que el sistema siga fallando en favor de un determinado sector. El impacto de la investigación es positivo, de ahí que su realización contribuye al dinamismo del sector manufacturero de la provincia del Guayas, avizorando nuevos ideales y perspectivas a ser consideradas para que optimizar su incursión en la contratación pública del país.

Mantilla, Ruíz, Mayorga, y Vilcacundo (2014) consideran que: "la competitividad del mundo actual exige que las empresas sean más productivas, sin importar su tamaño, para que estas puedan sobrevivir en los mercados” (p.17). En este sentido, ellas deben ser capaces de acoplarse a los cambios en conjunto con la innovación, investigación e incursión de las nuevas tecnologías.

Ante aquello, el proceso de innovación ya sea en la fase de proceso, producción y organización determinan los niveles de productividad y competitividad de una empresa, por lo que estos indicadores deben ser considerados como elementales dentro del contexto empresarial, comercial e industrial.

Por otra parte, la Organización para la Cooperación y el Desarrollo Económico-OCDE (2012) considera que: "el grado en que, bajo un conjunto de condiciones de libre mercado, un país puede ser capaz de producir bienes y servicios que superen el examen de la competencia internacional” (p.3). Es 
por ello, que las Pymes en la economía de los países juega un rol significativo, muestra de aquello es la situación en Colombia donde representan el 96\% de las empresas de la nación, la misma que genera el 66\% del empleo en el sector industrial, encargada de realizar el $25 \%$ de las exportaciones de carácter no tradicional, responsable de pagar el $50 \%$ de los salarios (Ministerio de Desarrollo, 2013).

En Ecuador, la productividad del sector manufacturero ha cobrado gran significancia durante los últimos años, tal es así que, provincias como Tungurahua se han proyectado como zonas ideal para la instalación de las industrias y empresas generadoras de productos y servicios distribuidos a nivel local, regional y nacional. De la misma manera, Ayala (2018) vicepresidente de la Cámara de Industrias de Guayaquil menciona que: “entre 2007 y 2016, la actividad industrial de Guayas se incrementó un 10,2\% por año, al margen de la desaceleración económica que se evidenció a escala nacional en 2016” (p.12).

Bajo este contexto, el crecimiento nacional al cierre del 2017 evidencia que alrededor de 32.866 empresas se encontraban domiciliadas en la provincia del Guayas, lo que representa el $41 \%$ de la red empresarial del Ecuador, seguido por Pichincha con 25.995 industrias. Los principales cantones costeros independiente de Guayaquil donde se registró un aumento significativo fueron Daule con el 21,4\% de crecimiento y 332 empresas; Durán, con el 12,8\% y 667 empresas y Samborondón que creció el 12,4\% con un total de 1.370 compañías (Ayala, 2018).

Cabe mencionar que, lo manifestado por el representante de la Cámaras de Industrias de Guayaquil evidencian que las empresas que encabezan la lista se refiere a las dedicadas a la producción de alimentos, seguidas por aquellas de reparación e instalación de maquinarias y equipos. De la misma manera, la sub rama industrial que refleja mayor presencia en la provincia del Guayas son las de producción de plásticos, con un margen de 70 empresas; la industria gráfica con 117; fabricación de prendas de vestir con 106; reparación de maquinarias con 89; producción de fármacos con 86, entre otras (García, 2018).

La potencialidad productiva de las Pymes de la provincia del Guayas se ve reflejada en el aporte al Producto Interno Bruto (PIB) con el 27,7\% por lo que se resalta que dentro del aspecto industrial es la que mayor desarrollo posee por su crecimiento del $10,2 \%$ en tiempos con donde la economía del país no era favorable.

En base al Estudio Sectorial: Panorama de la Inversión Empresarial en el Ecuador 2013-2017 realizado por Camino, Bermúdez, y Álvarado (2018) se demuestra que, a pesar de que la región de la sierra mantiene una mayor inversión empresarial con 11 provincias, así como un aporte anual del 52,1\%; la región de la costa, con 6 provincias genera anualmente un 44\% durante el período antes mencionado. En consecuencia, Guayas registró un monto de 
inversión referente a las constituciones y domiciliaciones por USD 318 millones, donde Guayaquil, Samborondón, Eloy Alfaro aportan con aproximadamente USD 214 millones, USD 80 millones y USD 19 millones respectivamente.

En concordancia con lo manifestado en el estudio, es evidente que la industria manufacturera durante el período 2016 recuperan su protagonismo con una participación activa que va con el $21 \%$ del total de las inversiones, por lo que el $72 \%$ del capital invertido en este componente se concentra en cuatro sectores económicos específicos: Industrias Manufactureras con el $28 \%$, Comercio (21\%, Actividades financieras y seguros (11\%), Agricultura (7\%) y Explotación de minas y canteras (6\%), mientras que el resto de sectores acumulan el $28 \%$ del total.

En el caso de la provincia del Guayas, el mayor monto de inversión se registra en el período 2015, con USD 881 millones de los cuales 767 millones corresponden a la ciudad de Guayaquil, donde el aumento de capital se concentra específicamente en industrias manufactureras, tales como: Industrias Lácteas Toni S.A. y el sector de comercio con la empresa “Leimagencorp S.A.”(Camino, Bermúdez, \& Álvarado, 2018).

\section{HIPÓTESIS (H1)}

La implementación a través de la normativa estipulada para el efecto y con la administración como organismo ejecutor de este sistema, ha influido determinada en el desarrollo económico y financiero del país al impulsar una mejor distribución de adjudicaciones de contratos entre las personas, permitiendo la participación de nuevos actores económicos en los concursos para adquisiciones de bienes y servicios logrando generar ahorros monetarios a las instituciones del estado, mejorando la calidad del gasto público.

La Contratación Pública incide de manera significativa en la producción de las empresas pymes manufactureras de la Provincia del Guayas.

\section{HIPÓTESIS NULA (H0)}

La Contratación Pública no incide de manera significativa en la producción de las empresas pymes manufactureras de la Provincia del Guayas.

\section{HIPÓTESIS ESPECÍFICAS}

- Las normativas de contratación públicas inciden de manera significativa en la producción de las pymes manufactureras de la provincia de la Guayas.

- Los proveedores inciden de forma significativa en la producción de las pymes de la provincia del Guayas. 


\section{METODOLOGÍA}

La metodología aplicada en el estudio, se basa en un diseño de corte longitudinal con enfoque cuantitativo, y es de naturaleza no experimental, además es de tipo exploratorio-descriptivo.

Entre las técnicas utilizadas para la recolección de datos se encuentra la hermenéutica, la misma que permite hacer un análisis documental de estudios realizados con antelación, sumándose a ella la triangulación de ideas, donde el autor puede exponer su experiencia y hacer conjeturas sobre lo manifestado por otros autores en relación al tema estudiado, teniendo presente que los trabajos citados guardan relación y congruencia con la relevancia de las Pymes en el dinamismo de la economía y desarrollo de los países. Además, por su aporte a la generación de empleo donde se origina lo positivo de la contratación pública de manera igualitaria y equitativa, especialmente para el sector manufacturero.

La economía de las empresas y la Dirección Estratégica, como una disciplina científica específica de esta ciencia, requieren de metodologías de investigación para analizar los fenómenos empresariales objetos de estudio que sean capaces de recoger toda su complejidad. En determinadas circunstancias se hacen necesarias metodologías que combinen información de distintas naturalezas: cuantitativa y cualitativa, subjetiva y objetiva, interna o externa al propio fenómeno estudiado.

De conformidad con el desarrollo utilizado para estructurar la investigación se aplicó una metodología con un enfoque mixto (Cualitativocuantitativo). Ante aquello, la metodología cualitativa podría entenderse como "una categoría de diseños de investigación que extraen descripciones a partir de observaciones que adoptan la forma de entrevistas, narraciones, notas de campo, grabaciones, transcripciones de audio y video, casetes, registros escritos de todo tipo, fotografías o películas y artefactos” (Herrera, 2008).

De la misma manera, Reguera (2008) la metodología cuantitativa es "exigible en la medición y control de variables, entendidas estas últimas como los aspectos o características observables y cuantificables de nuestro objeto de estudio". Por otra parte, es considerada como correlacional, ya que se utilizara para identificar el comportamiento, actitudes y reacciones de las personas frente a la situación que viven las empresas, para así poder combinar ciertos criterios de clasificación que ayuden a ordenar, agrupar y sistematizar los objetivos involucrados en el estudio de este problema.

Criterios de exclusión

En concordancia con lo manifestado, la provincia del Guayas en el 2018 registró un total de 1510 empresas manufactureras, pero dentro del estudio, como primer eje se separan las consideradas económicamente como PYMES, es decir, el número se reduce a solo el $30 \%$ de dicha cantidad inicial al considerarse sólo 453 de estas dentro del rango de estudio. 


\section{Criterios de inclusión}

Las empresas manufactureras que han cumplido con los requerimientos, elementos y certificaciones para ingresar al catálogo de proveedores del Estado, tema directo de la investigación se encuentran conformadas por un total de 176 Pymes manufactureras.

Se cuenta con una población finita claramente establecida por tanto se puede utilizar la fórmula para toma de muestra de sujetos de estudio que permita definir el número de elementos que se consideran para el estudio.

El tamaño de la muestra de las empresas manufactureras que participan en el sistema de contratación pública, se calculó mediante la siguiente fórmula:

$$
n_{1}=\frac{Z^{2} P Q N}{\left.Z^{2} P Q+E^{2}(N-1)\right)}
$$

Dónde:

$$
\begin{array}{ll}
\mathrm{Z}= & \text { Nivel de confianza al 95\% }(1,96) \\
\mathrm{P}= & \text { Probabilidad de éxito (cuando es desconocida } \mathrm{P}=0.5) \\
\mathrm{Q}= & \text { Probabilidad de fracaso o desconocimiento }(\mathrm{Q}=1-\mathrm{P}) \\
\mathrm{E}= & \text { Margen de error }(5 \%) \\
\mathrm{N}= & \text { Tamaño de la población }
\end{array}
$$

Reemplazando valores, obtenemos: $\mathrm{n}=80$

Instrumentos de investigación

Para la presente investigación se consideró la encuesta como herramienta para recolección de información con la finalidad de obtener los datos que permita realizar la comprobación de la hipótesis planteada en la presente investigación.

Presentación y análisis de resultados

Las Pymes se consideran como el eslabón más representativo en las actividades productivas de un país, estimado como fuentes generadoras de empleo y oferentes o demandantes de bienes y servicios. A pesar de aquello, existen barreras que limitan el emprendimiento de nuevos elementos productivos debido a múltiples factores, entre los que se encuentran el alto costo de financiamiento, incorporación de nuevas tecnologías generadoras de economía a gran escala, donde los costos de producción son minimizados convirtiéndolas en el motor dinamizador de la economía nacional.

Cabe mencionar que, el acceso de las Pymes al Sistema de Contratación Pública representa el 90\% de las empresas en América Latina y el Caribe, donde su aporte es relevante desde las perspectivas de empleo y productividad, debido a la inyección directa de recursos en la economía de los pueblos, incentivando las actividades productivas, industriales y comerciales, así como de servicios, coadyuvando a innovar la tecnología y el desarrollo científico para proponer mejores servicios y/o productos. 
Para dar inicio al proceso de análisis, es necesario tomar en consideración la definición de proveedor para el estado ecuatoriano, considerado como toda persona natural o jurídica, nacional o extranjera, o asociación de éstas, que se encuentren debidamente registradas en el Registro Único de Proveedores (RUP), así como de su habilitación para poder proveer bienes, ejecutar obras y prestar servicios, incluyéndose en estas actividades las de consultoría para el correcto y eficiente funcionamiento de las entidades gubernamentales (LNCP, 2008).

Para, Zúñiga Rendón (2011, pág. 28) la historia ecuatoriana se ha visto marcada por una serie de actos relacionadas a la corrupción en la administración pública, donde se ha demostrado la desviación de los recursos a registros de arcas particulares, sobornos a servidores públicos, sistemas judiciales, escaso control de veeduría, entre otros.

Debido a esto, en Ecuador, antes de que se expida la LOSNCP, el proceso de contratación pública era realizado por medio de la Ley de Contratación Pública y la Ley de Consultoría, sumándose a ellos los Reglamentos Internos de Contratación que emitían las entidades contratantes, los mismos que se caracterizaban por su diversidad de patrones, requisitos y márgenes de preferencia, situación que conllevaba a que cada entidad maneje los procesos de contratación de manera distinta dificultando los procesos de contratación y participación ciudadana realizada por medio de las veedurías (Castro Cuenca \& García López, 2012).

En aquel entonces no existía un Plan anual de Contrataciones que normalice de manera anticipada los procesos de contratación, situación que derivaba a las entidades estatales a desgastar los recursos económicos asignados para su funcionamiento, situación que ocasionaba el deterioro de los servicios públicos.

De la misma manera, no existía un Registro Único de Proveedores (RUP) a nivel nacional, donde cada una de las entidades manejaba sus propios procesos de calificación, ocasionando que se creen bases de datos de proveedores preferentes, lo que atentaba con los principios de igualdad de oportunidades de la participación para el sector productivo conformado por las Pymes. Además el uso de la tecnología en dicho proceso era indiferente, debido a que las invitaciones a los diversos concursos se los realizaban de manera física.

Otro factor que se pudo evidenciar como hecho referencial de la Contratación Pública antes de la LOSNCP, eran los gastos incurridos por las entidades contratantes, quienes tenían la obligación de realizar procesos de publicación en los diversos medios de comunicación del país; y, los proveedores, quienes debían cancelar costos originados por los Términos de Referencias de carácter obligatorio como uno de los requisitos para poder ser objeto de participación y licitación. 
No obstante, a partir del 2008, se procede a la expedición de la Ley Orgánica del Sistema Nacional de Contratación Pública, la misma que implementó un proceso de transformación en la manera en que se manejaba la contratación. Su proceso de legalización se dio con la publicación en el registro Oficial No. 395 del 4 de agosto de 2008, dando paso a una fase de innovación tecnológica; incentivo productivo a las empresas nacionales; planificación y correcto uso de los recursos públicos, entre otros.

Ante aquello, es evidente su variante a través del tiempo, el mismo que se plasma en la siguiente tabla:

Tabla 1. Registro Único de Proveedores

\begin{tabular}{|c|r|r|r|r|r|r|r|}
\hline Año & \multicolumn{1}{|c|}{2009} & \multicolumn{1}{c|}{2010} & \multicolumn{1}{c|}{2011} & \multicolumn{1}{c|}{2012} & \multicolumn{1}{c|}{2013} & \multicolumn{1}{c|}{2014} & \multicolumn{1}{c|}{ Total } \\
\hline Micro & 39.277 & 23.394 & 17.867 & 16.846 & 17.248 & 16.865 & 131.497 \\
\hline Pequeña & 6.650 & 3.237 & 2.822 & 3.012 & 2.491 & 1.598 & 19.810 \\
\hline Mediana & 1.471 & 661 & 562 & 559 & 457 & 345 & 4.055 \\
\hline Grande & 542 & 261 & 245 & 252 & 222 & 161 & 1.683 \\
\hline $\begin{array}{c}\text { No } \\
\text { definido }\end{array}$ & 107 & 54 & 51 & 37 & 45 & 39 & 333 \\
\hline TOTAL & 50.056 & 29.617 & 23.558 & 22.718 & 22.476 & 21.022 & 157.378 \\
\hline
\end{tabular}

Fuente: Sistema Nacional de Compras Públicas

Elaborado por: Autora, 2018.

En consecuencia, las compras públicas en el Ecuador representan el 30\% del gasto total, así como el 12\% del PIB, a pesar de aquello, este sistema no logra consolidar sus objetivos planteados con antelación, muy aparte a las políticas estatales implementadas para potenciar su efectividad y eficacia, con relaciona al 2014. Porque en el 2018 el Guayas acapara el 24 \% de la contratación, lo que indica que la provincia aumento en un 75 \% su compra pública.

Según el informe presentado por el Servicio Nacional de Contratación pública (SERCOP, 2017) considerada como la organización que lidera y regula la gestión transparente y eficiente de la contratación pública pone en manifiesto la información estadística hasta agosto del 2017, donde se pondera los principales resultados de la contratación pública ecuatoriana (Ver Tabla 2 y Gráfico 1).

Tabla 2. Participación de la contratación pública

\begin{tabular}{|c|c|c|c|}
\hline ARO & $\begin{array}{l}\text { CONTRATACION } \\
\text { PUBLICA } \\
\text { (CP) }\end{array}$ & $\begin{array}{l}\text { PRE SUPUESTO GENERAL } \\
\text { DEL ESTADO"* } \\
\text { (PGE INICIAL) }\end{array}$ & $\begin{array}{l}\text { PRODUCTO INTERNO } \\
\text { BRUTO": } \\
\text { (PIB NOMILAL) }\end{array}$ \\
\hline 2010 & $7.053,2$ & $21.282,1$ & $69.555,4$ \\
\hline 2011 & 9.857 .4 & 23.950 .2 & 79.276 .7 \\
\hline 2012 & 9.887 .5 & 26.109 .3 & 87.924 .5 \\
\hline 2013 & 10.842 .8 & 26.109 .3 & 95.129 .7 \\
\hline 2014 & 8.197 .6 & 34.300 .6 & 1022923 \\
\hline 2015 & 7.412 .5 & $36.317,1$ & 100.176 .8 \\
\hline 2016 & 6.916 .4 & 29.835 .1 & 96.217 .9 \\
\hline $\begin{array}{l}\text { Acumulado a agosto } \\
2017^{*}\end{array}$ & $3.318,9$ & $19.890,1$ & $67.242,1$ \\
\hline
\end{tabular}

Fuente: SERCOP, 2017 
Gráfico 1. Porcentaje de la participación de la contratación pública

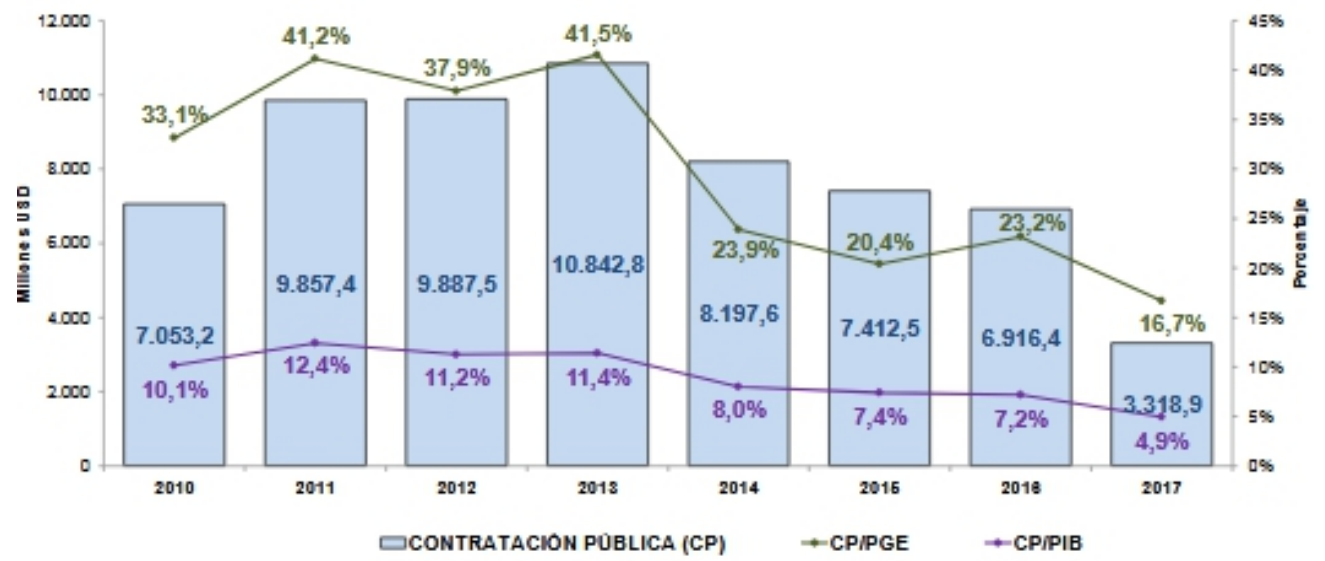

Fuente: SERCOP, 2017

De la misma manera, se puede evidenciar, que hasta el mes de agosto del 2017 se logró una adjudicación de 178 millones de dólares a diversos actores de la economía popular y solidaria (AEPS), en un 52,7\% más que el período 2016. Donde las microempresas proveedoras representaron alrededor del 85\% de un total de 3.713 AEPS que participaron en las diversas licitaciones públicas hasta el mes indicado con antelación. Entre ellas fueron los de tamaño pequeño y mediano, las mismas que demostraron un incremento de 139,5\% y 116,0\% respectivamente (Ver Tabla 2 y Gráfico 2).

Tabla 3. Número de proveedores de la economía popular y solidaria con sus respectivos montos adjudicados

\begin{tabular}{|c|c|c|c|c|c|c|}
\hline \multirow{2}{*}{ TAMANO DEL PROVEEDOR } & \multicolumn{2}{|c|}{ NÚMERO DE PROVEEDORES } & \multirow{2}{*}{ EVOLUCIÓN ANUAL } & \multicolumn{2}{|c|}{$\begin{array}{l}\text { MONTO ADJUDICADO } \\
\text { (Millones de dólares) }\end{array}$} & \multirow{2}{*}{$\begin{array}{l}\text { EVOLUCIÓN } \\
\text { ANUAL }\end{array}$} \\
\hline & AAGOSTO 2016 & AAGOSTO 2017* & & AAGOSTO 2016 & AAGOSTO 2017* & \\
\hline GRANDE & 6 & 4 & $-33,3 \%$ & 0,4 & 0,7 & $75,0 \%$ \\
\hline MEDIANA & 25 & 34 & $36,0 \%$ & 8,1 & 17,5 & $116,0 \%$ \\
\hline PEQUENAA & 349 & 537 & $53,9 \%$ & 36,5 & 87,4 & $139.5 \%$ \\
\hline \begin{tabular}{|l|} 
MICRO \\
\end{tabular} & 3.289 & 3.138 & $-4,6 \%$ & 71,6 & 72,4 & $1,1 \%$ \\
\hline TOTAL & 3.669 & 3.713 & $1,2 \%$ & 116,6 & 178,0 & $52,7 \%$ \\
\hline
\end{tabular}

Fuente: SERCOP, 2017 
Gráfico 2. Principales rubros adjudicados a AEPS hasta agosto 2017

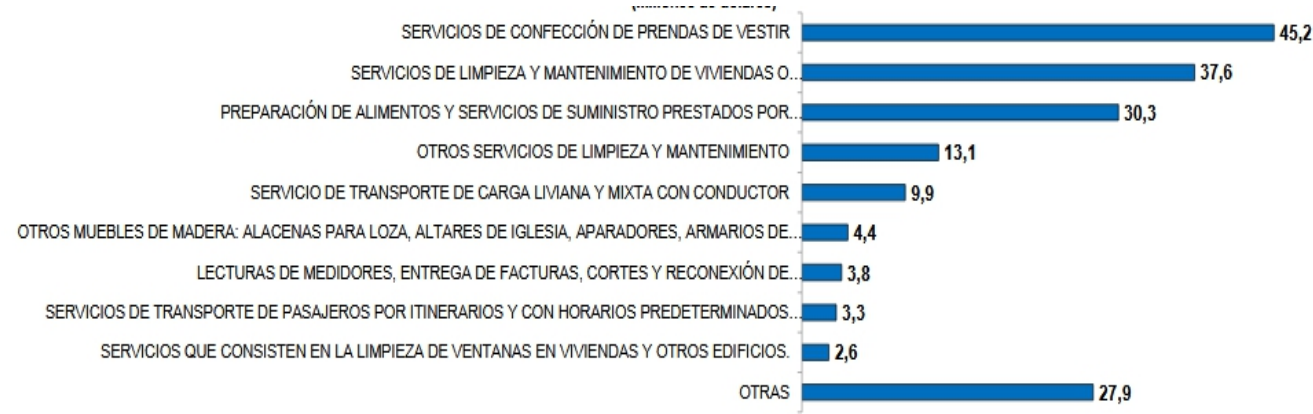

Fuente: SERCOP, 2017

\section{Análisis de datos}

Luego de haber realizado la investigación pertinente sobre el tema, se determina que el instrumento de investigación tuvo un grado de significancia de 0.01 , es decir que las preguntas realizadas arrojaron los datos con un valor de confianza por sobre el 0,5.

A continuación se indica la correlación que se halla entre la contratación pública y la producción de las empresa manufactureras de la provincia del guayas que constan en el catálogo de servicios del sistema de contratación pública.

Tabla 4. Correlación de variables

\section{Correlaciones}

\begin{tabular}{|cc|r|r|}
\hline & & CONT. PUB. & PRODUCC. \\
\hline CONT. & Correlación de Pearson & 1 &, $968^{* *}$ \\
PUBLICA & Sig. (bilateral) & &, 000 \\
& $\mathrm{~N}$ & 80 & 80 \\
\hline PRODUCC. & Correlación de Pearson &, $968^{* *}$ & 1 \\
& Sig. (bilateral) &, 000 & \\
& $\mathrm{~N}$ & 80 & 80 \\
\hline
\end{tabular}

**. La correlación es significativa en el nivel 0,01 (bilateral).

La información obtenida permite establecer que la contratación pública tiene incidencia significativa en el proceso de producción de las empresas manufactureras de la provincia del Guayas. Bajo este análisis se puede discernir que las empresas de las características estudiadas tienen un incremento significativo en su mantenimiento como organización, es también importante subrayar que los sujetos de estudio permiten definir que las 
empresas que entran al catálogo de servicios del Estado, tienden a forjar relaciones comerciales más fuertes en el sector privado tanto de manera interna y externa.

Por medio de un análisis particular se revisa las correlaciones que se encontraron entre cada una de las variables:

Correlación entre la VI (contratación pública) y VD1 (producción de pymes manufactureras).

Analizado los datos obtenidos, nos permite encontrar que la relación entre los dos aspectos estudiados y su propia incidencia fortalece la hipótesis de la importancia que la contratación pública tiene para el crecimiento de la producción de las empresas manufactureras de tal modo que esta herramienta se vuelve confiable a un grado que nos permite sostener el motivo de la investigación.

Tabla 5. Prueba de hipótesis

\section{Correlaciones}

\begin{tabular}{|cc|r|r|}
\hline & & Contratación & Producción \\
\hline Contratación & Correlación de Pearson & 1 &, $858^{* *}$ \\
& Sig. (bilateral) & &, 000 \\
& $\mathrm{~N}$ & 80 & 80 \\
\hline Crecimiento & Correlación de Pearson &, $858^{* *}$ & 1 \\
& Sig. (bilateral) &, 000 & \\
$\mathrm{~N}$ & 80 & 80 \\
\hline
\end{tabular}

**. La correlación es significativa en el nivel 0,01 (bilateral).

\section{Confiabilidad (Alpha de Cronbach)}

Luego de analizado las variables de esta investigación es importante demostrar la confiabilidad de la herramienta que fue utilizada para hallar la respuesta a la interrogante planteada, bajo ese enunciado se considera el Alpha de Cronbach para determinar el grado de confiabilidad de los elementos utilizados:

Tabla 6. Correlación de variables

Resumen de procesamiento de casos

\begin{tabular}{|c|c|c|c|}
\hline & & $\mathrm{N}$ & $\%$ \\
\hline \multirow[t]{3}{*}{ Casos } & Válido & 80 & 100,0 \\
\hline & Excluido $^{a}$ & 0 & 0 \\
\hline & Total & 80 & 100,0 \\
\hline
\end{tabular}

a. La eliminación por lista se basa en todas las variables del procedimiento. 
Tabla 7. Estadística de fiabilidad

Estadísticas de fiabilidad

\begin{tabular}{|r|r|r|}
\hline Alfa de Cronbach & $\begin{array}{c}\text { Alfa de Cronbach basada en elementos } \\
\text { estandarizados }\end{array}$ & N de elementos \\
\hline, 983 &, 984 & 2 \\
\hline
\end{tabular}

Gráfico 3. Correlación de variables

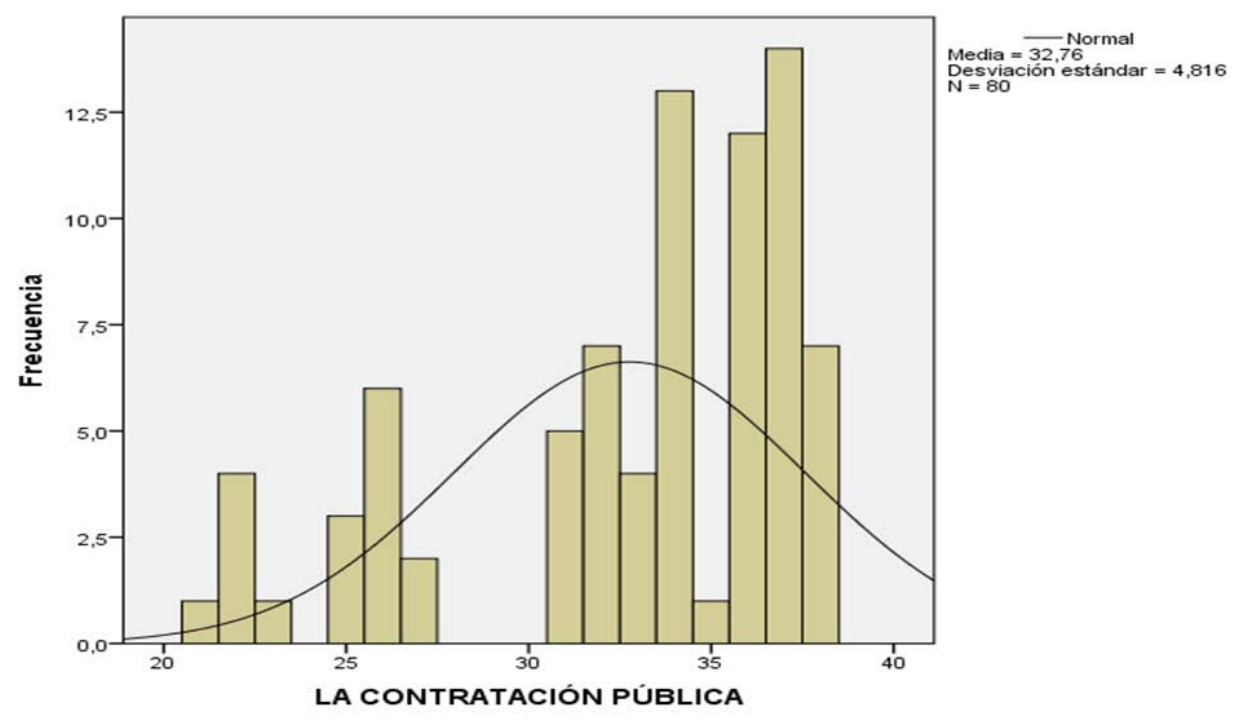

La tendencia proyectiva nos permite enfocarnos a un claro simétrico entre la contratación pública y su crecimiento a las empresas que entran en su entorno, según se pude observar el respectivo gráfico, enfocado en la muestra de 80 y se obtuvo una media de 32,76 con la desviación estándar de 4,816.

\section{Conclusión}

Con las mejoras sustanciales en el Sistema Nacional de Contratación Pública se ha logrado consolidar el crecimiento sostenible de la transaccionalidad, la innovación continua de nuevas utilidades, mayor nivel de inclusión de los actores económicos y ahorro significativo en el presupuesto inicial de compra.

En este sentido, a partir del año 2012 el Instituto Nacional de Contratación Pública-INCOP actualmente denominado SERCOP (Servicio Nacional de Contratación Pública) promovió el desarrollo de herramientas que permiten simplificar, publicitar y monitorear con un mejor grado de eficacia el ámbito de las compras gubernamentales. Por ello, se estableció los módulos de ejecución contractual en referencia a la ejecución de la obra, proceso de adquisición de bienes y contratación de servicios, para de esta manera cerrar el ciclo de las compras públicas; además las utilidades de transparentar los 
debidos procedimientos de contratación bajo la figura de un régimen especial, realización de ferias inclusivas y la constitución del catálogo artesanal, elementos indispensables que facilita el funcionamiento efectivo del Portal como una herramienta neutral en las transacciones públicas (Instituto Nacional de Contratación Pública, 2013, pág. 10).

En concordancia con lo expuesto, la contratación pública ha tenido un gran impacto en la economía ecuatoriana. Por tal motivo, en el período 2012 las cifras asignadas a la CP fue de USD 9.887.510.408 millones, que representó al Presupuesto General del Estado-PGE USD 26.109.270.276 millones; y al Producto Interno Bruto-PIB USD 87.924544.000 millones. En este sentido, el ahorro generado por la CP al Estado fue de USD 573 millones referente al ahorro anual, mientras que en el acumulado se obtuvo USD 1.064 millones (SERCOP, 2017, p. 20).

Durante el período de Producción de las Pymes Manufactureras en el años 2012 presentó alrededor de USD 26.117 millones superando las cifras obtenidas en el 2011 que fueron de USD 19.423 millones, con una diferencia significativa de USD 6.694 millones.

Previo a la certificación de competencias como operadores del sistema nacional de contratación pública de las entidades contratantes, por parte del SERCOP al personal responsable del manejo de la contratación pública se recomienda aplicar cursos de capacitación sobre la normativa jurídica que rige a la PYMES, así como también al personal del sector manufacturero, quienes deben conocer, manejar y dominar las obligaciones, derechos, beneficios y condiciones inherentes a las actividades productivas de las PYMES. Por esta razón, su actuar dentro de los procesos de compras públicas se ajuste a la normativa vigente protegiendo los derechos constitucionales de las personas, de las instituciones, de la administración pública y el correcto uso de los fondos y bienes públicos.

En este sentido, las normativas consideran un trato justo, lo que conlleva a que todo proveedor del sector manufacturero oferte sus obras, bienes y servicios y contraten con el Estado en iguales condiciones.

\section{References:}

1. Aguirre, R.J. (2016). Contratación pública ecuatoriana en el acuerdo comercial con la Unión Europea. Revista de Derecho, UASB.

2. Ayala, M. (28 de mayo de 2018). El 41\% de las industrias del país se desarrolla en Guayas. Obtenido de Cámara de Industrias de Guayaquil:

https://www.eltelegrafo.com.ec/noticias/economia/4/industriasdesarrollo-guayas-ecuador 
3. Camino, S., Bermúdez, N., \& Álvarado, E. (2018). Estudio Sectorial: Panorama de la Inversión Empresarial en el Ecuador 2013-2017. Quito: Dirección Nacional de Investigación y Estudios.

4. Díaz, C.J. (2016). El presupuesto referencial como un elemento esencial para afrontar la crisis económica del Ecuador en las compras públicas. Revista de Derecho Foro, 85.

5. Dirección Nacional de Estudios (2014). Servicio Nacional de Contratación Pública (SERCOP). Quito: SERCOP.

6. Fernández de Soto, J. (2014). Modelo Integral de Productividad, aspectos importantes para su implementación. Redalyc, 110-119.

7. García, E. (2018). Economía del país se incrementa. Obtenido de Diario El Telégrafo Digital: https://www.eltelegrafo.com.ec/noticias/economia/4/industriasdesarrollo-guayas-ecuador

8. González Tamayo, J. (2016). El régimen ecuatoriano de contratación pública: entre el proteccionismo y la apertura comercial. revista de Derechos, 3.

9. González, J. (2013). Estudio sobre la competitividad de Pymes incubadas en Empreser de México A.C. sucursal, San Quintín. Universidad Autónoma de Baja California.

10. Herrera. (2008). Metodología Cualitativa.

11. Ibarra Cisneros, M., González Torres, L., \& Dummer Flores, M. (2017). Competitividad empresarial de las pequeñas y medianas empresas manufactureras de Baja California. Estudios fronterizos, 4560.

12. Instituto Nacional de Contratación Pública. (2013). Rendición de cuentas 2012. Informe 2012 INCOP, 10.

13. Instituto Nacional de Estadística y Censo. (2017). Reporte del Índice de Producción de la Industria Manufacturera (IPI-M) - Mayo 2017. Quito: INEC.

14. Jácome, H., \& King, K. (2013). Estudios Insdustriales de la micro, pequeña y mediana empresa. Flacso, 15.

15. López, N., \& Martínez, F. (2012). Innovación, Pymes y Globalización. México: Académica Española.

16. Mantilla, L., Ruíz, M., Mayorga, C., \& Vilcacundo, A. (2014). La competitividad de las Pymes manufactureras de Ambato-Ecuador. Universidad de Cartagena, 17-30.

17. Medina, J. (2012). El Modelo Integral de Productividad. Bogotá: Fondo de Publicaciones Universidad Sergio Arboled.

18. Murán, J. (2012). Manual de calidad. Editorial Reverte.

19. Organización Mundial del Comercio (2016). Acuerdo sobre Contratación Públic. Obtenido de Organización Mundial del 
Comercio:

https://www.wto.org/spanish/tratop_s/gproc_s/gp_gpa_s.htm

20. Paz \& Miño (2015). Historia de los impuestos en Ecuador. Quito: SRIPUCE-THE.

21. Peña, S. (2016). Análisis del Marco Legal de Compras püblicas en Ecuador: Oprtunidades y barreras para la incorporación de criterios sostenibles. Quito: PNUMA.

22. Pérez Uribe, R. (2015). ¿Por qué fracasan las pymes en Colombia? Revista de Economía, 12.

23. ProMéxico (2013). Pymes, eslabón fundamental para el crecimiento en México. Negocios Internacionales, 50-66.

24. Reguera (2008). metodología Cuantitativa.

25. Rivas, L., \& López, N. (2010). Interrogantes y respuestas sobre la nueva Ley de Contratación Pública. Quito: Unidad Tecnológica América.

26. Ron, R., \& Sacoto, V. (2015). Las PYMES ecuatorianas: su impacto en el empleo como contribución del PIB PYMES al PIB total. revista espacios, 15.

27. Rosagel, S. (2012). Pequeña empresa, líderes en exportación. Revista manufactura, 78.

28. Rozenwurcel, G. \& Drewes, L. (2012). unsam.edu.ar.

29. Servicio Nacional de Contratación Pública . (2015). Sociedad Española de Directivos de la Salud: Marco Regulatorio Europeo en Contratación Pública. Quito: SERCOP.

30. SR Obando Torresano. (2011). dspace.ups.edu.ec.

31. Vázquez, Y., \& Arredondo, L. (2014). Importancia de las pymes en el mundo. Revista Cubana de Economía Internacional, 23.

32. Yance Carvajal, C., Solís Granda, L., Burgos Villamar, I., \& Hermidia Hermidia, L. (2017). La importancia de las Pymes en el Ecuador. Observatorio de Economía Latiniamericana, 89.

33. Zúñiga Rendón, C. (2011). La Contratación Pública en el Ecuador antes de la creación de la Ley Orgánica del Sistema Nacional de Contratación Pública, LOSNCP. Revista Jurídica. 